\title{
Development of an adaptable headspace sampling method for metabolic profiling of the fungal volatome
}

DOI:

10.1039/C8AN00841H

\section{Document Version}

Accepted author manuscript

Link to publication record in Manchester Research Explorer

\section{Citation for published version (APA):}

Ahmed, W. M., Geranios, P., White, I. R., Bromley, M., Lawal, O., Nijsen, T., Goodacre, R., Read, N., \& Fowler, S. J. (2018). Development of an adaptable headspace sampling method for metabolic profiling of the fungal volatome. The Analyst. https://doi.org/10.1039/C8AN00841H

\section{Published in:}

The Analyst

\section{Citing this paper}

Please note that where the full-text provided on Manchester Research Explorer is the Author Accepted Manuscript or Proof version this may differ from the final Published version. If citing, it is advised that you check and use the publisher's definitive version.

\section{General rights}

Copyright and moral rights for the publications made accessible in the Research Explorer are retained by the authors and/or other copyright owners and it is a condition of accessing publications that users recognise and abide by the legal requirements associated with these rights.

\section{Takedown policy}

If you believe that this document breaches copyright please refer to the University of Manchester's Takedown Procedures [http://man.ac.uk/04Y6Bo] or contact uml.scholarlycommunications@manchester.ac.uk providing relevant details, so we can investigate your claim.

\section{OPEN ACCESS}




\section{Analyst}

\section{Accepted Manuscript}

This article can be cited before page numbers have been issued, to do this please use: W. M. Ahmed, P. Geranios, I. R. White, M. Bromley, O. Lawal, T. Nijsen, R. Goodacre, N. Read and S. J. Fowler, Analyst, 2018, DOI: 10.1039/C8AN00841H.

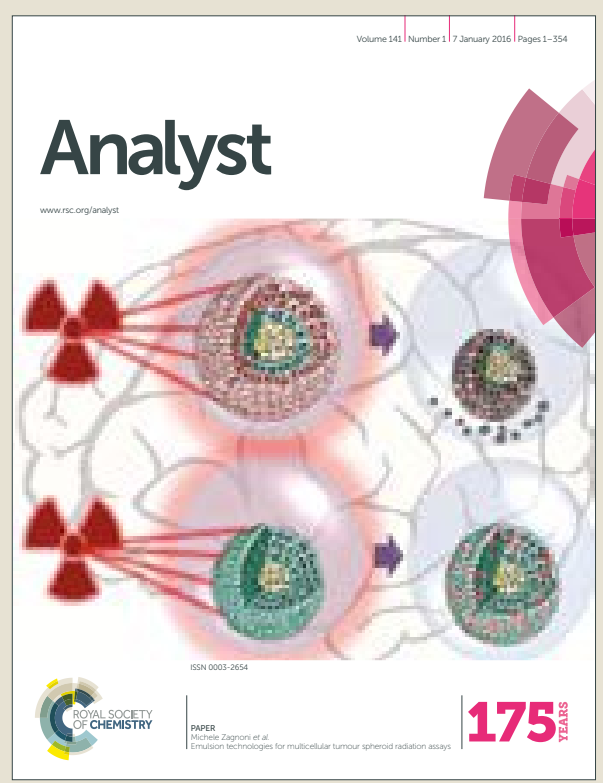

This is an Accepted Manuscript, which has been through the Royal Society of Chemistry peer review process and has been accepted for publication.

Accepted Manuscripts are published online shortly after acceptance, before technical editing, formatting and proof reading. Using this free service, authors can make their results available to the community, in citable form, before we publish the edited article. We will replace this Accepted Manuscript with the edited and formatted Advance Article as soon as it is available.

You can find more information about Accepted Manuscripts in the author guidelines.

Please note that technical editing may introduce minor changes to the text and/or graphics, which may alter content. The journal's standard Terms \& Conditions and the ethical guidelines, outlined in our author and reviewer resource centre, still apply. In no event shall the Royal Society of Chemistry be held responsible for any errors or omissions in this Accepted Manuscript or any consequences arising from the use of any information it contains. 


\title{
Development of an adaptable headspace sampling method for metabolic profiling of the fungal volatome
}

Waqar M. Ahmed ${ }^{1}{ }^{*}$, Pavlos Geranios ${ }^{1}$, Iain R. White ${ }^{2}$, Oluwasola Lawal ${ }^{1}$, Tamara M. Nijsen ${ }^{3}$, Michael J. Bromley ${ }^{1}$, Royston Goodacre ${ }^{2}$, Nick D. Read ${ }^{1}$, Stephen J. Fowler ${ }^{1,4 \#}$

1. School of Biological Sciences, University of Manchester, UK

2. School of Chemistry, Manchester Institute of Biotechnology, University of Manchester, UK

3. Philips Research, Royal Philips B.V., The Netherlands

4. Manchester Academic Health Science Centre, Manchester University Hospitals NHS Foundation Trust, UK

${ }^{*}$ Equal authorship

\# Corresponding author: Stephen.Fowler@manchester.ac.uk

\begin{abstract}
Pulmonary aspergillosis can cause serious complications in people with a suppressed immune system. Volatile metabolites emitted by Aspergillus spp. have shown promise for early detection of pathogenicity. However, volatile profiles require further research, as effective headspace analysis methods are required for extended chemical coverage of the volatome; in terms of both very volatile and semi-volatile compounds. In this study, we describe a novel adaptable sampling method in which fungal headspace samples can be sampled continuously throughout a defined time period using both active (pumped) and passive (diffusive) methods, with the capability for samples to be stored for later off-line analysis. For this method we utilise thermal desorption-gas chromatographymass spectrometry to generate volatile metabolic profiles using Aspergillus fumigatus as the model organism. Several known fungal-specific volatiles associated with secondary metabolite biosynthesis (including $\alpha$-pinene, camphene, limonene, and several sesquiterpenes) were identified. A comparison between the wild-type $A$. fumigatus with a phosphopantetheinyl transferase null mutant strain $(\triangle p p t A)$ that is compromised in secondary metabolite synthesis, revealed reduced production of sesquiterpenes. We also showed the lack of terpene compounds production during the early growth phase, whilst pyrazines were identified in both early and late growth phases. We have demonstrated that the fungal volatome is dynamic and it is therefore critically necessary to
\end{abstract}


sample the headspace across several time periods using a combination of active and passive sampling techniques to analyse and understand this dynamism.

\section{Introduction}

Pulmonary aspergillosis (PA) is a group of fungal infections of the respiratory tract which can exacerbate disease in patients with ineffective immune defences, such as those with cystic fibrosis or asthma ${ }^{1}$. The causative pathogens of PA (whether invasive or non-invasive forms) are Aspergillus spp. for which the current clinical identification method requires microbial culture or PCR which can be time consuming (up to several days), inefficient, and insensitive. Delays in initiating treatment for these diseases can have a significant outcome on patient mortality ${ }^{2}$. Early and accurate detection of Aspergillus spp. colonisation within the lung is therefore crucial for diagnosis of PA.

Volatile organic compounds (VOCs) are known to play a role in intracellular communication between microbes, and between microbes and the host ${ }^{3,4}$, and are largely investigated as potential biomarkers of respiratory disease and for the differentiation of pathogens ${ }^{5,6}$. Exploratory analysis of VOCs emitted by fungi typically involves headspace analysis of in vitro cultures, as the headspace gas is a rich source of VOCs and directly indicative of extracellular activity. However, the headspace can be sensitive to several factors including secondary metabolite production ${ }^{7}$, interaction with other fungi or bacteria ${ }^{8,9}$, environmental contaminants or stresses ${ }^{10}$, and sampling and analysis methods ${ }^{11}$. It is therefore important to profile the volatome under a range of conditions, such as at different growth phases, using a suitable sampling method.

In this study we have used $A$. fumigatus CEA10 as the model organism to develop an adaptable and simple headspace sampling method in which samples may be taken at several time points from the same culture, sampled under active (pumped) or passive (diffusive) sampling means, and where VOCs may be stored and transported for off-line analysis. To demonstrate proof-of-principle of the 
method we developed, we tested the method's performance by identification of known fungal VOCs from A. fumigatus and compared the VOC profile of wild-type A. fumigatus to a mutant strain lacking polyketide synthase (PKS) and non-ribosomal-peptide synthase (NRPS) activity and hence with impaired secondary metabolism production.

\section{Experimental}

\subsection{Strains and growth conditions}

Two strains of Aspergillus fumigatus were used for headspace analysis - a wild-type strain (FGSC A1163) and a phosphopantetheinyl transferase (PptA) null mutant ( $\triangle \mathrm{pptA})$ derived from the aforementioned wild-type. The $\Delta$ pptA strain was recently shown to lack effective secondary metabolite, siderophore, and lysine biosynthesis, ${ }^{12}$ rendering the enzyme a potential antifungal target.

A. fumigatus CEA10 $(n=5)$ and $\Delta \operatorname{pptA}(n=6)$ strains were cultured on Sabouraud Dextrose Agar (Oxoid, Basingstoke, UK) for three to five days at $37^{\circ} \mathrm{C}$. Spores (conidia) were harvested with $10 \mathrm{~mL}$ PBS-Tween80 (Sigma-Aldrich, Gillingham, UK) $0.05 \%$ v/v (PBS composition in 1 litre: $\mathrm{NaCl}$ 8g, KCl 0.2 $\left.\mathrm{g}, \mathrm{Na}_{2} \mathrm{PO}_{4} 1.44 \mathrm{~g}, \mathrm{KH}_{2} \mathrm{PO}_{4} 0.24 \mathrm{~g}, \mathrm{pH}=7.4\right)$. The suspension was subsequently filtered through a sterile double layer of Miracloth (Merck Millipore, Darmstadt, Germany), and the filtrate was centrifuged at 5,500 $\mathrm{g}$ for $10 \mathrm{~min}$. After discarding the supernatant, the precipitate containing the spores was re-suspended in $5 \mathrm{~mL}$ of fresh PBS-Tween $800.05 \%$. Spores were counted using a FuchsRosenthal haemocytometer and a conventional optical benchtop microscope to optimise the concentration of spores.

\subsection{Headspace sampling}

The headspace chamber was composed of glass, and the flask opening was sealed with a PTFE stopper (Sigma-Aldrich, Gillingham, UK). A hollow stainless-steel tube was exposed within the headspace chamber, and VOC samples were trapped on a sorbent tube fixed onto a three-way 
stainless-steel ball valve (Thames Restek, Saunderton, UK) attached to the outside part of the exposed hollow tube. This set up prevented any aerosolised conidia to attach outside of the sample sorbent tube and within the valve. PTFE tape was used as a seal and to prevent fixtures prone to gas leaks.

For the headspace sampling, $100 \mathrm{~mL}$ Büchner filter flasks (Scientific Glass Laboratories Limited, Stoke-on-Trent, UK) containing $10 \mathrm{~mL}$ of solid Aspergillus minimal media (AMM) were inoculated with $1 \mathrm{~mL}$ of conidial suspension containing a total of $3 \times 10^{5}$ spores. After gentle shaking to ensure the suspension has covered the entirety of the media surface, the flasks were sealed and incubated at $37^{\circ} \mathrm{C}$. All manipulations were carried out in a Class 2 microbiological cabinet under aseptic conditions.

During active sampling, headspace gas was drawn out using a low-flow pump (Acti-VOC, Markes International, Llantrisant, UK) with a flow rate of $50 \mathrm{~mL} / \mathrm{min}$ for $2 \mathrm{~min}$, resulting in a total sample volume of $100 \mathrm{~mL}$ per sample. Headspace was sampled at two time points for germination (0 to $8 \mathrm{~h})$ and maturation or conidiation (12 to $24 \mathrm{~h}$ ) for all fungal strains and media samples. Passive sampling (i.e. diffusive sampling) was performed between 0 and $8 \mathrm{~h}$, and 12 and $24 \mathrm{~h}$. Passive sampling relied upon reaching a pressure-dependant equilibrium; i.e., between the atmospheric pressure within the headspace chamber and the external atmospheric pressure. Headspace gas is drawn out of the chamber with minimal flow (generated by increased pressure), where VOCs are adsorbed onto the sorbent material. The total volume was therefore unknown as the passive flow per minute was not recorded. Although purging the headspace was an option, we chose not to use a gas purge method (i.e. with inert gases such as $\mathrm{CO}_{2}$ and $\mathrm{N}_{2}$ ) as induction may disrupt the culture (by drastically lowering the $\mathrm{pH}$, through $\mathrm{HCO}_{3}{ }^{-}$in the case of $\mathrm{CO}_{2}$ ) and cause turbulence within the headspace chamber. An illustration of active and passive sampling is shown in Figure 1.

After sampling, flasks were treated with Chemgene HLD 4 D (Star Lab group, Milton Keynes, UK) for 24 h. After heating to bring the contained media to a melting point, the media was discarded, the flasks 
2

3

4

5

6

7

8

were thoroughly washed with water, rinsed with sterile water and autoclaved to be used for subsequent sampling experiments.
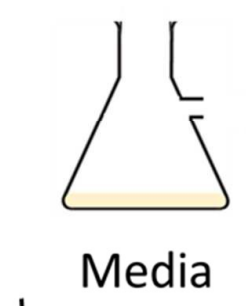

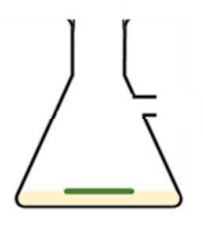

CEA10

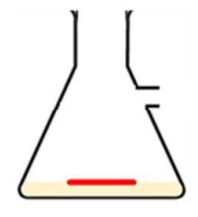

$\triangle P p t A$
Passive sampling a

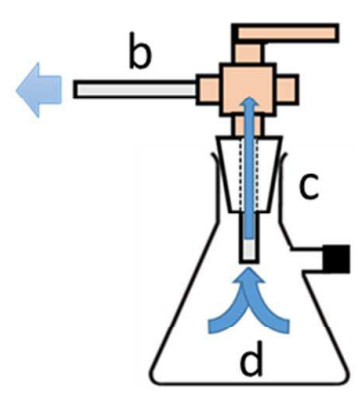

Active sampling

a

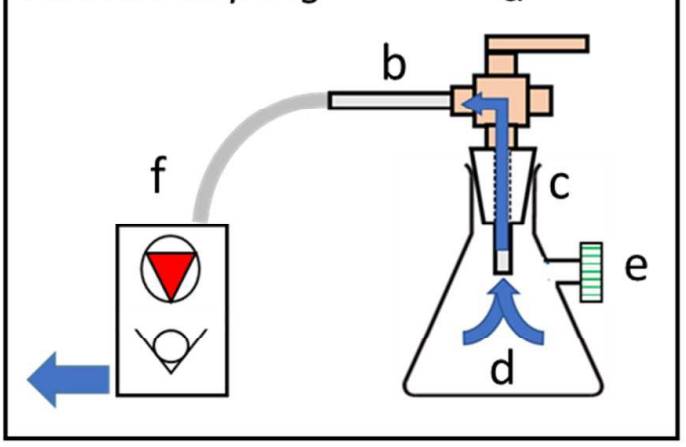

Figure 1. Illustration of culture samples (media, CEA10, and $\triangle p p t A)$, and headspace sampling methods (passive sampling and active sampling) where the blue arrows signify headspace gas flow. The headspace sampling apparatus is comprised of several parts, labelled as a) three way ball valve (brass or stainless steel), b) thermal desorption tube containing sorbent material, c) hollow tube (PTFE or stainless steel) and PTFE stopper combination forming an gas tight seal, d) headspace chamber (glass), e) input flow filter (in active sampling mode only), and f) a calibrated and controlled low flow pump.

\subsection{Sample desorption and analysis}

Samples were purged through stainless-steel thermal desorption tubes packed with a multi-bed sorbent containing Tenax TA and Carbograph 5TD (Markes International, Llantrisant, UK). The sorbent material was chosen based on the extended trapping range $\left(C_{3}\right.$ to $\left.C_{30}\right)$ without retaining a high concentration of water molecules. 
Tubes were reconditioned at $330{ }^{\circ} \mathrm{C}$ for 60 min with a constant dry $\mathrm{N}_{2}$ flow of $100 \mathrm{~mL} / \mathrm{min}$ through the tubes. Sorbent tubes were tightly sealed with brass caps during storage and transportation. After adsorption, sorbent tubes were transported for analysis in an airtight container and stored at $4^{\circ} \mathrm{C}$ for no longer than one week before desorption.

Samples were analysed in randomised blocks by Thermal Desorption-Gas Chromatography-time of flight-Mass Spectrometry (TD-GC-tof-MS). Briefly, tubes were heated in a thermal desorption unit (Unity 2, Markes International, Llantrisant, UK) to $320^{\circ} \mathrm{C}$ (5 min hold) before being purged with helium ( $1 \mathrm{~mL} / \mathrm{min})$. Compounds were transferred onto a cold trap (kept at $0{ }^{\circ} \mathrm{C}$ ) before being desorbed a second time to $330^{\circ} \mathrm{C}$ ( 3 min hold) and transferred into the GC column for separation. The Agilent DB-5ms Ultra Inert GC column (30 m length, 0.25 mm internal diameter, $0.25 \mu \mathrm{m}$ film thickness) within an Agilent 6890 GC oven (Agilent Technologies, West Lothian, UK) had a helium carrier gas flow set to a constant pressure method ( $69 \mathrm{kPa})$, and was subjected to a temperature ramped program starting at $40{ }^{\circ} \mathrm{C}$, first ramp to $170{ }^{\circ} \mathrm{C}$ at $6{ }^{\circ} \mathrm{C} / \mathrm{min}$, and the second ramp to $250{ }^{\circ} \mathrm{C}$ at $15^{\circ} \mathrm{C} / \mathrm{min}$ (total GC run time of $27 \mathrm{~min}$ ). After chromatographic separation, VOCs were transferred to the mass spectrometer (GCT Premier, Waters Corporation, Manchester, UK) and subjected to electron ionisation (EI) in positive mode $(70 \mathrm{eV})$, before further separation in the time-of-flight mass analyser. Mass spectra were acquired in centroid format at a scan rate of $10 \mathrm{scans} / \mathrm{s}$, between $\mathrm{m} / \mathrm{z}$ 40 and 500.

Each sample was purged with an internal standard to account for analytical variation and to normalise samples (1 ppmv 4-bromofluorobenzene in $\mathrm{N}_{2}$ ), and each batch run was bracketed by a mixture of VOC standard chemicals (> $95 \%$ purity, Sigma-Aldrich, Gillingham, UK) to monitor instrument performance. External standards were also run independently to confirm the identification of some VOCs of interest to MSI level $1^{13,14}$ (see Table S1 for further information on the VOC mixture and external standards).

\subsection{Data analysis and VOC Identification}


Raw GC-MS data files were converted to mzXML format using the MSConvert software

(ProteoWizard v. 3.0.8738, Los Angeles, USA). Samples were pre-processed (peak integration and smoothing, baseline correction, and retention time alignment) using both the $X C M S$ package ${ }^{15}$ through R (R Core Development team, version 3.4.2) and Chromalynx batch analysis using the ApexTrack peak integration algorithm (Masslynx software, Waters Corporation, Manchester, UK). XCMS and Chromalynx parameters were tuned using the VOC standards mixture (Table S1). The National Institute of Standards and Technology mass spectral library (NIST version 2014, Gaithersburg, USA) was used for compound identification of chromatographic peaks i.e. MSI level 2. In addition, some VOCs were identified with NIST and a chemical standard i.e. MSI level 1 (where two orthogonal data are used to identify a compound) $)^{13,14}$.

Peak intensities were normalised by the internal standard for each sample. To test statistical significance between two different sample groups, the non-parametric Mann Whitney-U test was used $(\alpha \leq 0.05)$. For multivariate analysis, features with $>50 \%$ of missing values were removed. Remaining missing values were imputed using the random forest proximity algorithm ${ }^{16,17}$. Features were then range scaled and mean centred.

Principal component analysis (PCA) between strains and time points was carried out to visualise sample separation, batch effects, and identify outliers. If no clear separation was visible between groups, further supervised analysis in the form of Principal component-discriminant function analysis (PC-DFA) was performed using the adegenet package ${ }^{18}$. Here, the number of PCs used in the final model were optimised using stratified random sampling (x 1000) where data were split into an internal training and validation set (ratio of 75:25). From this internal validation, the optimised number of PCs with the lowest root mean squared error (RMSE) from the percentage of successful predicted outcomes was chosen for the final model. PC-DFA loadings from each discriminant function were extracted and features were identified through a NIST search. The features and 
identifications were compared to the results of compounds identified through the Chromalynx software.

\section{Results}

\subsection{Headspace sampling method development}

Data pre-processing and treatment resulted in a total of 1450 features $(\mathrm{m} / \mathrm{z}$ fragment ions at a specific retention time), which served as input into multivariate analysis. Clusters of discriminatory features were extracted and a total of 35 compounds were identified, 11 of which were identified to MSI level 1 i.e. with a NIST library search and chemical standards).

Headspace sampling was performed to assess the impact of different hardware materials on volatile profiles obtained from tissue culture flasks. The selected materials were glass for the chamber, stainless-steel to channel VOCs towards the sorbent trap, and polytetrafluoroethylene (PTFE) material to seal flask openings. Chromatograms of headspace samples from an empty plastic cell culture flask compared to an empty glass flask are shown in the supplementary information (Figure S1), where a larger number of contaminant VOCs were present from the cell culture flask.

Sampling was performed within the incubator to minimise changes in temperature and pressure, where any fluctuation in these would alter the vapour pressure of headspace VOCs. The sampling volume was chosen based on headspace volume clearance. In our case, this was set to $50 \mathrm{~mL} / \mathrm{min}$ for $2 \mathrm{~min}$ to clear a headspace volume of approximately $80 \mathrm{~mL}$. Headspace gas was replaced by ambient air through the flask side arm. Active sampling resulted in increased number of VOCs identified, the most significant being sesquiterpenes, when compared to passive sampling (see chromatograms in Figure S2).

From blank control samples of the apparatus, known VOCs associated with instrument artefacts (i.e. from the analytical instrument, sorbent tubes, cleaning and maintenance, and headspace apparatus) were identified as difluoro(dimethyl)silane (1.65 $\mathrm{min})$, ethanol (1.77 $\mathrm{min})$, benzene (2.49 $\mathrm{min})$, 
benzoic acid (13.24 $\mathrm{min}$ ) and several siloxane compounds throughout the retention time range which, through process of elimination, were thought to originate from the borosilicate glass flask (cyclotrisiloxane, hexamethyl-, cyclotetrasiloxane, octamethyl-, cyclopentasiloxane, decamethyl-, cycloheptasiloxane, tetradecamethyl-). VOCs from AMM media control samples were identified as acetic acid (2.07 $\mathrm{min})$, benzaldehyde (7.55 $\mathrm{min})$, phenol (7.88 $\mathrm{min})$, acetophenone (10.21 $\mathrm{min})$, nonanal (11.21 min), decanal (13.83 $\mathrm{min})$, 2-ethylhexyl acrylate (14.32 $\mathrm{min})$, and several saturated alkanes $\left(C_{12}-C_{16}\right)$. A typical chromatogram of a media-only control sample is shown in supplementary Figure S2. These compounds were excluded from further analysis for the identification of discriminatory fungal VOCs.

\subsection{Fungal VOC profiles}

The sum of all peak intensities i.e. the total ion count was assessed for whole sample reproducibility between replicate headspace samples for each strain. Across CEA10 replicates, passive sampling showed an increased relative standard deviation (RSD) between active $(0-8 \mathrm{~h}=13.82 \%, 12-24 \mathrm{~h}=$ $14.13 \%)$ and passive sampling $(0-8 \mathrm{~h}=19.66 \%, 12-24 \mathrm{~h}=19.24 \%)$, whereas $\Delta$ pptA samples showed higher variation between replicates overall compared to that CEA10 for all both active $(0-8 \mathrm{~h}=36.03$ $\%, 12-24 h=28.60 \%)$ and passive $(0-8 h=18.06 \%, 12-24 h=50.93 \%)$ sampling methods, except $8 \mathrm{~h}$ passively collected samples (CEA10 = $19.66 \%, \Delta p p t A=18.06 \%)$.

No clear class separation was shown by PCA. Figure 2 shows a PC-DFA scores plot between wild-type CEA10 and mutant $\triangle$ pptA strains (6 PCs, Total Explained Variance: $82.3 \%$ ) for both passive and active sampling methods (total of four classes: CEA10 active, CEA10 passive, $\Delta \mathrm{pptA}$ active, and $\Delta \mathrm{pptA}$ passive). PC-DFA loadings from the first discriminant function (DF1, Figure S3) revealed VOCs unique to CEA10, identified as cyclopentene, 2-methoxyfuran, $\alpha$-bergamotene, and himachalene. Additional sesquiterpene VOCs were identified in A. fumigatus CEA10 and included isocaryophillene and $\alpha$ bisabolene from one CEA10 strain sample. Differential VOCs from DF1 were also identified for $\Delta p p t A$, identified as 2,5-dimethylfuran, 3-methylbutanal, styrene, and dodecanoic acid. In addition, a 
sesquiterpene identified as cis-thujopsene was identified from one $\Delta p p t A$ strain sample. VOCs $(n=4)$ were also identified in at least one sample from both strains, namely ethyl acetate, $\alpha$-pinene, camphene, and limonene.

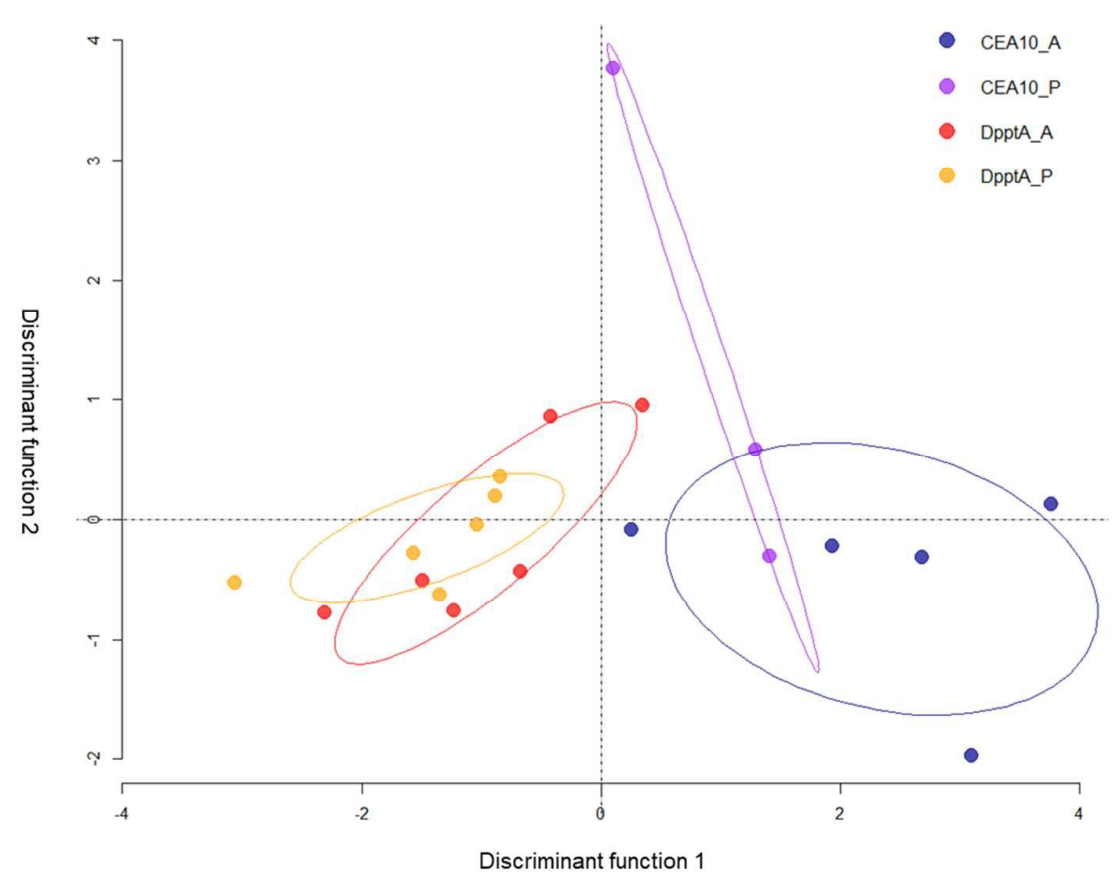

Figure 2. PC-DFA scores plot (nPCs: 6, TEV: 82.3\%) of A. fumigatus CEA10 (CEA10-active in blue, and CEA10-passive in purple) and $\triangle p p t A$ strains (ApptA-active in red, and $\triangle p p t A$-passive in orange) sampled from 12 to $24 \mathrm{~h}$. Ellipses are drawn as a guide and are have no statistical significance.

Eleven VOCs were identified across a minimum of three replicates (in either or both CEA10 and $\Delta \mathrm{pptA}$ strains from the 12 to $24 \mathrm{~h}$ time point using active sampling) and are listed in Table 1 , along with mean peak intensities and $p$ value following statistical testing between CEA10 and $\triangle \mathrm{pptA}$ sample groups.

Table 1. Fungal volatile organic compounds identified from CEA1O and $\triangle p p t A$ strains.

55
56
57
58
59
60




\subsection{Volatiles specific to germinating and maturing colonies}

PC-DFA was also carried out on headspace samples (10 PCs, TEV: $93.5 \%$ ) collected between 0 to $8 \mathrm{~h}$ (i.e. during germination) and between 12 to $24 \mathrm{~h}$ time periods (Figure 3 ) using both active and passive sampling techniques, hence resulting in four classes used in the model. No fungal VOCs were found for samples collected passively between 0 and $8 \mathrm{~h}$, whereas using active sampling at $8 \mathrm{~h}$ contained methylpyrazine and pyrazine. These compounds were also present in 12 to $24 \mathrm{~h}$ passive and active samples with no difference between the two time-points for both pyrazine $(p=0.383)$ or methylpyrazine $(p=0.200)$. PC-DFA Loadings from DF2 revealed the presence of terpene-based compounds (a-pinene, camphene, limonene, and himachelene) in the 12 to $24 \mathrm{~h}$ samples. 


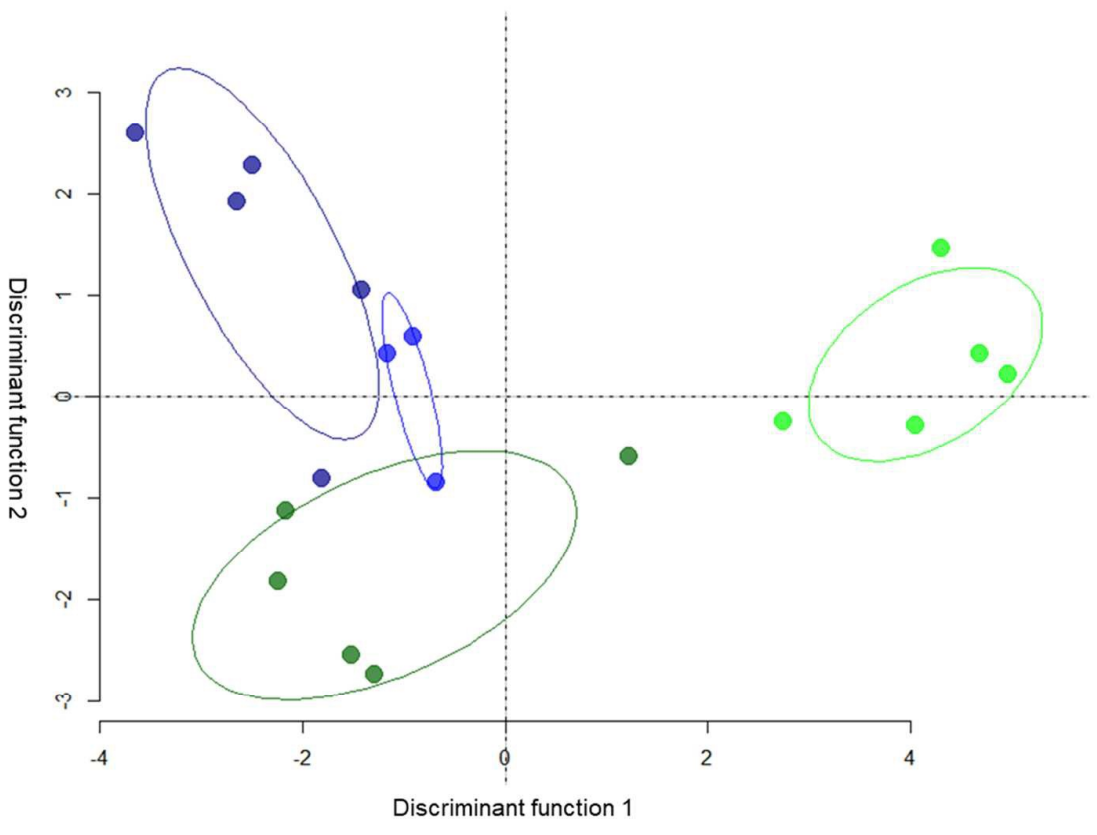

Figure 3. PC-DFA scores plot (nPCs: 10, TEV: $93.5 \%$ ) of A. fumigatus CEA10 headspace sampled at 0 to $8 \mathrm{~h}$, where samples collected passively are coloured light green and samples collected actively are coloured dark green. Also shown are samples collected between 12 to $24 \mathrm{~h}$, where samples collected passively are coloured light blue, and samples collected actively are coloured dark blue. Ellipses are drawn as a guide and are have no statistical significance.

\section{Discussion}

We have developed a headspace sampling method for off-line analysis of the fungal volatome, allowing continuous sampling of a culture over several time-points with passive and/or active sampling methods. Materials were tested and chosen to minimise instrument artefacts and contaminants. As thermal desorption tubes are used to pre-concentrate headspace VOCs, storage and transport of samples between laboratories and off-line analysis by TD-GC-MS is possible. In addition, the sorbent material, flask volume, and gas flow can be changed. Together with metabolomic-driven data processing and supervised analysis, a time segmented non-targeted profile for an extended coverage of the fungal volatome is possible.

To test the method, we have demonstrated proof-of-concept through sampling and identification of known VOCs associated with $A$. fumigatus (Table 1 ), which included monoterpene compounds $\alpha$ pinene, camphene, and limonene identified in both $A$. fumigatus CEA10 and $\triangle$ pptA strains. The 
sesquiterpene himachalene was identified more frequently and in higher abundance in the wild-type strain CEA10 (MSI level 2) compared to other sesquiterpenes. Sampling metabolically-derived VOCs is generally difficult, and we have shown that some compounds were not identified across all replicates of a either CEA10 or $\triangle \mathrm{pptA}$ strains.

Monoterpenes and sesquiterpenes have been identified from $A$. fumigatus headspace by Heddergott et al. $^{19}$ and in a recent study investigating breath VOCs as diagnostic markers for invasive $\mathrm{PA}^{20}$. As described by in both studies, and through the online KEGG database ${ }^{21}$, biosynthesis of terpene-based VOCs originates from the melvanoate pathway, essential for the formation of secondary metabolites, in which farsenyl diphosphate and geranyl diphosphate are the precursors to the biosynthesis of monoterpenes and sesquiterpenes, respectively. The suppression of sesquiterpenes in the $\triangle p p t A$ mutant is consistent with the role of pptA in activating NRPS and PKS enzymes. Sesquiterpenes are precursors for the production of some secondary metabolites ${ }^{19}$. It is important to note that terpene compounds are not exclusive to Aspergillus spp. and may be released by other fungi species such as Candida. Terpenes are also common in plant-based diets - an important consideration for clinical biomarker studies.

Pyrazines are nitrogen-containing cyclic compounds and their presence in bacterial metabolism is well described ${ }^{22}$. Recently, pyrazine compounds have gained interest as fungal metabolites ${ }^{23}$. In our study, we were able to identify pyrazine and methylpyrazine in both the CEA10 and $\Delta$ pptA strains. The biosynthesis of pyrazines by Aspergillus spp. is not fully understood, however pyrazine groups can be found within the chemical structure of some secondary metabolites such as aspergillic acid or gliotoxin $^{19}$, in which pyrazines may be released in the headspace during their biosynthesis or degradation. As pyrazines share a similar chemical structure to azoles, they may have an inhibitory role in cellular growth. Styrene, another cyclic compound, was also identified from $\Delta p p t A$. Although styrene has been identified in other Aspergilli ${ }^{24}$, and to our knowledge this is the first description of it being identified in $A$. fumigatus. 
Ethyl acetate was identified only once in CEA10 and 5/6 $\Delta$ pptA samples, and has been identified from fungal headspace (including $A$. fumigatus) in a previous study ${ }^{25}$. 3-methylbutanal and decanoic acid were also more frequently identified in $\triangle p p t A$. 3-methylbutanal, and a methylated furan were identified in a recent study investigating fungal headspace profiles under hypoxia conditions ${ }^{10}$. Increased ethyl acetate, 3-methylbutanal, decanoic acid, and the identified furan-based compounds may be indicative of fatty acid biosynthetic intermediates in the $\Delta$ pptA strain, due to the inactivation of fatty acid synthases.

To demonstrate the versatility of the headspace sampling method, we identified VOCs released during the early growth phase of $A$. fumigatus, which included pyrazine and methylpyrazine using active and passive sampling techniques. As mentioned earlier, pyrazine compounds are known fungal VOCs. However, in many studies headspace samples were collected during a single time point and usually at a mature stage of growth (for example $48 \mathrm{~h}$ to $96 \mathrm{~h}$ ). It is therefore important to recognise that some VOCs are suggestive of early cellular growth, and therefore useful in the early indication or diagnosis of aspergillosis, as shown by previous studies ${ }^{10,26}$. Furthermore, VOCs can be transiently present within the headspace (depending on their partition coefficient) and sampling at different time periods is necessary to capture VOCs only present during a particular growth phase. For instance, in this study we showed the absence of terpene compounds from $0-8 \mathrm{~h}$, and their presence in 12-24 h. As terpene compounds are thought to be by-products from the production of secondary metabolites, they may be useful indicators of successful host colonisation or culture growth.

Several VOCs that have been reported in literature were not identified within our samples, for which there may be several reasons, such as the use of different strains, different culture conditions (e.g. incubation temperature, growth media) or different sampling time-points to those previously used. In addition, several VOCs were identified in the media (such as alkanes, aldehydes/ketones, and 
alcohols). These were excluded from further analysis as quantitative analysis would require a higher number of replicates to determine their increased or decreased level of production.

Alternative methods are available for headspace analysis, such as direct mass spectrometric analysis without chromatographic separation, but these do not allow resolution of isobaric chemical species. Techniques such as off-line SPME may be an alternative to this method ${ }^{19,27}$. Future work would involve comparing different sorbents and developing denatured headspace profiles of several species, and global metabolome coverage between both headspace and liquid matrices.

\section{Conclusions}

In this study we have demonstrated a novel proof-of-concept for the sampling and analysis of fungal culture headspace using $A$. fumigatus as the test organism. The presence of known fungal VOCs from acquired TD-GC-MS data shows feasibility of the method for profiling the in vitro volatome, towards a biological understanding of fungal metabolism. In addition, we have shown that volatile metabolites are indicative of germination, which has potential benefits for clinical diagnostics. Finally, We have shown the dynamic nature of the fungal volatome and how important it is to sample the fungal headspace at different times using active and passive sampling techniques to analyse and understand the significance of this dynamism.

\section{Acknowledgements}

PG was financially supported by the European Union grants PITN-GA-2013-607963 to NDR. WA and OL were financially supported by the European Union FP7 Marie Sklodowska-Curie Actions IAPP BreathDx-611951 to TMN, RG, and SJF. We would like to thank Craig Portsmouth, Maxim Wilkinson, and Kirti Vekaria for their support (University of Manchester).

\section{Responsibilities}


PG and WA contributed equally to this study. They both developed the sampling system, performed data processing, and wrote the first draft of the manuscript. PG carried out microbial culturing and headspace sample collection. WA, OL, and IRW developed the analytical and data processing methods. IRW performed sample analysis and sorbent tube conditioning. MJB provided the KO strain samples. MJB, TJN, RG, NDR, and SJF conceived the study, provided instruments and expertise, and revised the manuscript.

\section{Conflicts of interest}

The authors declare no conflicts of interest.

\section{References}

1 G. D. Brown, D. W. Denning, N. A. R. Gow, S. M. Levitz, M. G. Netea and T. C. White, Hidden killers: Human fungal infections, Sci. Transl. Med., 2012, 4, 1-10.

2 D. Kontoyiannis and G. Bodey, Invasive aspergillosis in 2002: An update, Eur. J. Clin. Microbiol. Infect. Dis., 2002, 21, 161-172.

3 L. J. Barkal, C. L. Procknow, Y. R. Álvarez-Garciá, M. Niu, J. A. Jiménez-Torres, R. A. BrockmanSchneider, J. E. Gern, L. C. Denlinger, A. B. Theberge, N. P. Keller, E. Berthier and D. J. Beebe, Microbial volatile communication in human organotypic lung models, Nat. Commun., , DOI:10.1038/s41467-017-01985-4.

4 P. Albuquerque and A. Casadevall, Quorum sensing in fungi a review, Med. Mycol., 2012, 50, 337-345.

5 L. D. Bos, P. J. Sterk and S. J. Fowler, Breathomics in the setting of asthma and chronic obstructive pulmonary disease, J. Allergy Clin. Immunol., 2016, 138, 970-976.

6 W. M. Ahmed, O. Lawal, T. M. Nijsen, R. Goodacre and S. J. Fowler, Exhaled Volatile Organic Compounds of Infection: A Systematic Review, ACS Infect. Dis., 2017, acsinfecdis.7b00088.

7 S. U. Morath, R. Hung and J. W. Bennett, Fungal volatile organic compounds: A review with emphasis on their biotechnological potential, Fungal Biol. Rev., 2012, 26, 73-83.

8 B. Briard, C. Heddergott and J. P. Latgé, Volatile compounds emitted by Pseudomonas aeruginosa stimulate growth of the fungal pathogen Aspergillus fumigatus, MBio, 2016, 7, 15.

9 A. H. Neerincx, B. P. Geurts, M. F. J. Habets, J. A. Booij, J. van Loon, J. J. Jansen, L. M. C. Buydens, J. van Ingen, J. W. Mouton, F. J. M. Harren, R. A. Wevers, P. J. F. M. Merkus, S. M. Cristescu and L. A. J. Kluijtmans, Identification of Pseudomonas aeruginosa and Aspergillus fumigatus mono- and co-cultures based on volatile biomarker combinations, J. Breath Res., 2016, 10, 16002. 
out the hypoxia volatile metabolic signature of Aspergillus fumigatus, J. Breath, 2017, 11, 36003.

11 J. F. Cavalli, X. Fernandez, L. Lizzani-Cuvelier and A. M. Loiseau, Comparison of Static Headspace, Headspace Solid Phase Microextraction, Headspace Sorptive Extraction, and Direct Thermal Desorption Techniques on Chemical Composition of French Olive Oils, J. Agric. Food Chem., 2003, 51, 7709-7716.

A. Johns, D. H. Scharf, F. Gsaller, H. Schmidt, T. Heinekamp, M. Straßburger, J. D. Oliver, M. Birch, N. Beckmann, K. S. Dobb, J. Gilsenan and B. Rash, A Nonredundant Phosphopantetheinyl Transferase, PptA, Is a Novel Antifungal Target That Directs Secondary Metabolite, Siderophore, and Lysine Biosynthesis in Aspergillus fumigatus and Is Critical for Pathogenicity, MBio, 2017, 8, 1-22.

L. W. Sumner, A. Amberg, D. Barrett, M. H. Beale, R. Beger, C. A. Daykin, T. W.-M. Fan, O. Fiehn, R. Goodacre, J. L. Griffin, T. Hankemeier, N. Hardy, J. Harnly, R. Higashi, J. Kopka, A. N. Lane, J. C. Lindon, P. Marriott, A. W. Nicholls, M. D. Reily, J. J. Thaden and M. R. Viant, Proposed minimum reporting standards for chemical analysis, Metabolomics, 2007, 3, 211221.

14 O. Fiehn, D. Robertson, J. Griffin, M. van der Werf, B. Nikolau, N. Morrison, L. W. Sumner, R. Goodacre, N. W. Hardy, C. Taylor, J. Fostel, B. Kristal, R. Kaddurah-Daouk, P. Mendes, B. van Ommen, J. C. Lindon and S.-A. Sansone, The metabolomics standards initiative (MSI), Metabolomics, 2007, 3, 175-178.

15 C. Smith, J. Elizabeth, G. O'Maille, R. Abagyan and G. Siuzdak, XCMS: processing mass spectrometry data for metabolite profiling using Nonlinear Peak Alignment,Matching,and Identification, ACS Publ., 2006, 78, 779-787.

P. S. Gromski, Y. Xu, H. L. Kotze, E. Correa, D. I. Ellis, E. G. Armitage, M. L. Turner and R. Goodacre, Influence of missing values substitutes on multivariate analysis of metabolomics data., Metabolites, 2014, 4, 433-52.

17 D. J. Stekhoven and P. Bühlmann, Missforest-Non-parametric missing value imputation for mixed-type data, Bioinformatics, 2012, 28, 112-118.

18 T. Jombart, Adegenet: A R package for the multivariate analysis of genetic markers, Bioinformatics, 2008, 24, 1403-1405.

19 C. Heddergott, A. M. Calvo and J. P. Latge, The Volatome of Aspergillus fumigatus, Eukaryot. Cell, 2014, 13, 1014-1025.

20 S. Koo, H. R. Thomas, S. D. Daniels, R. C. Lynch, S. M. Fortier, M. M. Shea, P. Rearden, J. C. Comolli, L. R. Baden and F. M. Marty, A Breath Fungal Secondary Metabolite Signature to Diagnose Invasive Aspergillosis., Clin. Infect. Dis., 2014, 59, 1733-1740.

21 H. Ogata, S. Goto, K. Sato, W. Fujibuchi, H. Bono and M. Kanehisa, KEGG: Kyoto encyclopedia of genes and genomes, Nucleic Acids Res. , 1999, 27, 29-34.

22 S. Schulz and J. S. Dickschat, Bacterial volatiles: the smell of small organisms., Nat. Prod. Rep., 2007, 24, 814-842.

23 J. S. Dickschat, Fungal volatiles - a survey from edible mushrooms to moulds, Nat. Prod. Rep., 2017, 34, 310-328. 
24 Z. Cheng, M. Li, P. J. Marriott, X. Zhang, S. Wang, J. Li and L. Ma, Chemometric analysis of the volatile compounds generated by aspergillus carbonarius strains isolated from grapes and dried vine fruits, Toxins (Basel)., 2018, 10, 1-17.

25 S. Matysik, O. Herbarth and A. Mueller, Determination of microbial volatile organic compounds (MVOCs) by passive sampling onto charcoal sorbents, Chemosphere, 2009, 76, 114-119.

26 M. Hertel, S. Hartwig, E. Schütte, B. Gillissen, R. Preissner, A. M. Schmidt-Westhausen, S. Paris, I. Kastner and S. Preissner, Identification of signature volatiles to discriminate Candida albicans, glabrata, krusei and tropicalis using gas chromatography and mass spectrometry, Mycoses, 2015, n/a-n/a.

27 C. P. Costa, D. Gonçalves Silva, A. Rudnitskaya, A. Almeida and S. M. Rocha, Shedding light on Aspergillus niger volatile exometabolome, Sci. Rep., 2016, 6, 27441. 